\title{
Phosphoproteomic analysis of electroacupuncture analgesia in an inflammatory pain rat model
}

\author{
SI-HYOUNG LEE ${ }^{1 *}$, SUN-YOUNG KIM $^{2 *}$, JI-HWAN KIM ${ }^{1}$, HYE-YUN JUNG $^{2}$, \\ JEONG-HEE MOON $^{2}$, KWANG-HEE BAE ${ }^{2}$ and BYUNG-TAE CHOI ${ }^{1}$ \\ ${ }^{1}$ Division of Meridian and Structural Medicine, School of Korean Medicine, Pusan National University, \\ Yangsan 626-870; ${ }^{2}$ Medical Proteomics Research Center, KRIBB, Daejeon 305-806, Republic of Korea
}

Received December 15, 2011; Accepted April 10, 2012

DOI: $10.3892 / \mathrm{mmr} .2012 .879$

\begin{abstract}
The phosphorylation changes of nociceptive signaling proteins in the spinal cord dorsal horn (SCDH) are important in creating exaggerated pain following peripheral inflammation. Electroacupuncture (EA) has been widely used to relieve acute and chronic inflammatory pain in human and experimental pain models. In the present study, we performed a phosphoproteomic analysis to investigate whether EA alters protein phosphorylation in SCDH to attenuate pain development. Inflammatory hyperalgesia was induced by intraplantar injection of complete Freund's adjuvant (CFA) into the rat hind paw. EA treatment at ST36 and SP6 acupoints alleviated thermal hyperalgesia of the CFA-induced inflammatory pain model rats. The SCDH proteins from the control, inflammatory pain model and EA treatment rats were separated by 2-dimensional gel electrophoresis and the alterations in phosphoproteins were detected by Pro-Q Diamond staining. Eight proteins were differentially phosphorylated following EA treatment in the inflammatory pain model. Aldolase C, nascent polypeptide-associated complex $\alpha$, stress-induced phosphoprotein 1 and heat shock protein 90 were identified as phosphoproteins whose expression was increased, whereas GDP dissociation inhibitor 1, thiamine triphosphatase, phosphoglycerate kinase 1 and 14-3-3 $\gamma$ were phosphoproteins whose expression was decreased. This is the first phosphoproteomic screening study to elucidate the working mechanisms
\end{abstract}

Correspondence to: Dr Byung-Tae Choi, Division of Meridian and Structural Medicine, School of Korean Medicine, Pusan National University, Yangsan 626-870, Republic of Korea

E-mail: choibt@pusan.ac.kr

Dr Kwang-Hee Bae, Medical Proteomics Research Center, Korea Research Institute of Bioscience and Biotechnology (KRIBB), 50 Eon-dong, Yusung-gu, Daejeon 305-806, Republic of Korea E-mail:khbae@kribb.re.kr

*Contributed equally

Key words: electroacupuncture, analgesia, phosphoproteomics, spinal cord, complete Freund's adjuvant of EA analgesia. The results suggest that the regulation of cellular pathways in which the identified proteins are involved may be associated with an EA analgesic mechanism.

\section{Introduction}

Peripheral inflammation frequently leads to the development of chronic pain, which is a leading cause of disability globally. In inflammatory pain, peripheral stimuli activate primary afferent neurons and are transmitted to secondary sensory neurons in the spinal cord dorsal horn (SCDH). Persistent noxious stimuli increase the excitability of secondary neurons in the SCDH and decrease the pain threshold. Early studies attributed this central sensitization to the facilitated synaptic transmission of nociceptive signals in the $\operatorname{SCDH}(1,2)$. Various signaling molecules such as glutamate receptors, protein kinase A (PKA), protein kinase C (PKC), phosphatidylinositol 3-kinase (PI3K), $\mathrm{Ca}^{2+}$-/calmodulin-dependent kinase II (CaMKII) and mitogen-activated protein kinases (MAPKs), including extracellular signal-regulated kinase (ERK), p38 and c-jun N-terminal kinase (JNK), have been shown to be involved in altered nociceptive processing in the SCDH (3-5). Activation of these molecules is capable of inducing signaling cascades that lead to the subsequent expression of pain-associated genes such as prodynorphin, neurokinin-1 (NK-1) and cyclooxygenase-2 (COX-2) (6,7).

Acupuncture, a traditional therapeutic method in oriental medicine, has long been clinically used to relieve pain. In particular, electroacupuncture (EA), where electrical stimulation is applied to acupuncture needles, is widely used in clinical and animal pain model studies. A number of EA studies have demonstrated that EA has analgesic effects on inflammatory pain models (8-10). The possible mechanisms of EA analgesia include activation of the endogenous opioid system and alteration of the nociceptive transmission pathways, which are associated with central sensitization in the SCDH (8-11). Although several proteins are known to be involved in EA analgesia, the underlying mechanisms of EA analgesia are not fully understood. In particular, considering that the phosphorylation changes of signaling proteins in the SCDH are important in inducing central sensitization, understanding how EA is capable of altering the phosphorylation state of signaling proteins in SCDH is crucial in order to elucidate the 
EA analgesic mechanism. Protein phosphorylation is a posttranslational modification that is involved in diverse biological phenomena (12). The reversible phosphorylation of serine, threonine and tyrosine residues is involved in the regulation of the functional status of proteins and peptides in eukaryotic organisms (13). An estimated $30 \%$ of cell proteins are likely to be phosphorylated, often at multiple sites, during their existence (14). Therefore, phosphoproteomic analysis offers an excellent potential for the identification of candidate regulatory proteins in various cell states.

The intraplantar injection of complete Freund's adjuvant (CFA) in the hind paw of rats is a commonly used model to study chronic inflammatory pain (15). In the present study, we performed a phosphoproteomic analysis of SCDH proteins from control, inflammatory pain model and EA treatment rats. Using Pro-Q Diamond staining and mass spectrometry (MS) analysis after 2-dimensional gel electrophoresis (2-DE), eight proteins differentially phosphorylated following EA treatment were identified in the inflammatory pain model. Information from this phosphoproteome profiling may help us to understand the altered phosphorylation pattern of proteins involved in EA analgesia, thereby increasing the overall understanding of the EA analgesic mechanism in the SCDH.

\section{Materials and methods}

Animals. Male Sprague-Dawley rats weighing approximately $200 \mathrm{~g}$ were obtained from Dooyeol Biolab (Korea). The rats were housed under constant environmental conditions at $22^{\circ} \mathrm{C}$ and a 12-h dark-light cycle with free access to food and water. Prior to experimental manipulation, rats were allowed to acclimatize to the housing facilities for 2 weeks. Experimental procedures and animal handling were conducted under the Animal Care Guidelines of the Animal Experimental Committee of Pusan National University.

CFA-induced inflammatory pain model. To develop the inflammatory pain model, $100 \mu \mathrm{l}$ of CFA solution $(1 \mathrm{mg} / \mathrm{ml}$ Mycobacterium tuberculosis; Sigma-Aldrich, St. Louis, MO, USA) was injected subcutaneously into the plantar side of the left hind paw of rats (16). Control rats received identical volumes of saline.

Experimental design. In the behavioral study, rats were divided into three groups ( $n=6$ per group): i) control group of rats injected with $100 \mu \mathrm{l}$ of saline intraplantarly; ii) inflammatory pain group of rats injected with $100 \mu \mathrm{l}$ of CFA intraplantarly; and iii) EA treatment group of rats injected with $100 \mu \mathrm{l}$ of CFA intraplantarly and treated with EA. After the behavioral study, three rats from each group were used for the phosphoproteomic study.

EA treatment. In the EA treatment group, rats were treated with EA once a day from the first CFA post-injection day for 2 days. Two pairs of $0.25 \mathrm{~mm}$-diameter stainless-steel acupuncture needles (Dong-Bang, Korea) were bilaterally inserted to the equivalent of human acupoint ST36 (Zusanli) and SP6 (Sanyinjiao) on the hind legs of rats. The needles at the ST36 and SP6 acupoints were connected to an A300 Pulsemaster electro-pulse generator (World Precision Instruments,
Sarasota, FL, USA). An electrical pulse with $2 \mathrm{~Hz}$ frequency and $1 \mathrm{~mA}$ was delivered for $20 \mathrm{~min}$.

Behavioral test. The rats from each group were tested 1 day prior to and 2 days after CFA or saline injection, respectively. Rats were individually placed on a heated $\left(56^{\circ} \mathrm{C}\right)$ platform, and then the paw withdrawal latency (PWL) time to the first sign of left paw licking or jumping to avoid heat pain was recorded with an automatic electronic timer (Harvard Apparatus, Holliston, MA, USA). A cut-off period of $20 \mathrm{sec}$ was set to avoid tissue injury.

Sample preparation for phosphoproteomic analysis. To investigate alternative protein phosphorylation changes in the $\mathrm{SCDH}$, the rats from three experimental groups ( $\mathrm{n}=3$ per group) were sacrificed under anesthesia following behavioral tests. The lumbar spinal cords at the level of L4-L5 were rapidly dissected and the dorsal specimens were obtained separately. The samples were immediately homogenized in buffer A [50 mM Tris- $\mathrm{HCl}$ (pH 7.1), $100 \mathrm{mM} \mathrm{KCl,} \mathrm{20 \%} \mathrm{glycerol} \mathrm{and}$ protease inhibitors] and sonicated for $1 \mathrm{~min}$. Subsequently, homogenates were centrifuged twice at 50,000 rpm (226,000 x g) for $1 \mathrm{~h}$ at $4^{\circ} \mathrm{C}$. The protein concentrations were measured in supernatant fractions using the Bradford assay.

Isoelectric focusing and electrophoresis. The spinal cord homogenate (150 $\mu \mathrm{g}$ of protein) was mixed with rehydration buffer [9 $\mathrm{M}$ urea, 4\% CHAPS, $2 \mathrm{M}$ thiourea, $40 \mathrm{mM}$ dithiothreitol (DTT) and 2\% immobilized pH gradient (IPG) buffer]. Protein samples were directly applied to IPG strips $(\mathrm{pH} 3-11$, $13 \mathrm{~cm}$ ) and rehydrated for $14 \mathrm{~h}$ at room temperature. Isoelectric focusing was performed using the Multiphor II apparatus (GE Healthcare, UK). The initial voltage was maintained at $300 \mathrm{~V}$ for $1 \mathrm{~min}$ and linearly increased from 300 to $3,500 \mathrm{~V}$ within $1.5 \mathrm{~h}$. The voltage was then maintained at $3,500 \mathrm{~V}$ for $8 \mathrm{~h}$. The plate temperature was kept constant at $25^{\circ} \mathrm{C}$ during isoelectric focusing. Focused IPG strips were briefly equilibrated for $15 \mathrm{~min}$ with equilibration solution [50 mM Tris- $\mathrm{HCl}(\mathrm{pH}$ 8.8), $6 \mathrm{M}$ urea, 2\% sodium dodecyl sulfate (SDS) and $30 \%$ glycerol] containing $1 \%$ DTT, and the strips were equilibrated again with the same solution containing 5\% iodoacetamide instead of DTT for $15 \mathrm{~min}$. Equilibrated strips were directly loaded onto $13 \%$ polyacrylamide gels $\left(150 \times 150 \times 1.5 \mathrm{~mm}^{3}\right)$ or stored at $-80^{\circ} \mathrm{C}$ until use. Polyacrylamide gels loaded with IPG strips were run constantly at $30 \mathrm{~mA}$ per gel with the Protean II $\mathrm{Xi} / \mathrm{XL}$ system (Bio-Rad, Hercules, CA, USA).

Staining and image analysis. For the detection of phosphoproteins, the gels were stained using a Pro-Q Diamond phosphoprotein gel stain kit (Molecular Probes, Grand Island, NY, USA) according to the manufacturer's instructions. Briefly, a 2-DE gel was fixed with $45 \%$ methanol and $5 \%$ acetic acid (400 $\mathrm{ml}$ per gel) for $2 \mathrm{~h}$. It was then washed three times with water (400 $\mathrm{ml}$ per gel). Gels were incubated in Pro-Q Diamond phosphoprotein stain solution (400 $\mathrm{ml}$ per gel) for $4 \mathrm{~h}$, and destained by washing four times in $50 \mathrm{mM}$ sodium acetate, $\mathrm{pH} 4.0$, and $4 \%$ acetonitrile (ACN; $400 \mathrm{ml}$ per gel) for $1 \mathrm{~h}$. Images were captured using a Typhoon 9410 apparatus (GE Healthcare) with an emission wavelength of $580 \mathrm{~nm}$. Following fluorescence scanning, the gels were stained again using a modified Coomassie brilliant blue-G250 method for 
visualization of the total expressed proteins and then scanned by the Power Look 2100XL scanner (Umax, Atlanta, GA, USA) using a film-scanning mode to obtain the stained images. The phosphoproteins were analyzed using the Samespot ${ }^{\mathrm{TM}}$ software (Nonlinear Dynamics, La Jolla, CA, USA). The protein spot volume was evaluated based on the lowest boundary mode of background selection. pI values were estimated based upon the pI range of the IPG strips used in the experiments. The experiments were independently conducted three times, and altered proteins, which were investigated by at least two independent experiments in triplicate, were selected as reliable data.

In-gel digestion and identification. Spots of interest were manually excised from 2-DE gels and destained with chemical reducers to remove silver. Briefly, $50-100 \mathrm{ml}$ of the freshly prepared reducing solution (1:1 mixture of $30 \mathrm{mM}$ potassium ferricyanide and $100 \mathrm{mM}$ sodium thiosulfate) was added to the gel plugs and mixed. After the brown color disappeared, the gel plugs were rinsed with water, and $200 \mathrm{mM}$ ammonium bicarbonate was added for $20 \mathrm{~min}$. Subsequently, the gel plugs were cut into small sections, washed with water and dehydrated repeatedly with ACN until the sections turned opaque white. Gel sections were then dried in a vacuum centrifuge for $30 \mathrm{~min}$, and the proteins were digested with $20 \mathrm{ng} / \mathrm{ml}$ of sequencing grade-modified trypsin (Promega, Madison, WI, USA) for $16-24 \mathrm{~h}$ at $37^{\circ} \mathrm{C}$.

Digested peptides were extracted using extraction solution [50\% ACN and 5\% trifluoroacetic acid (TFA)], and the extracted peptides were dried using a vacuum drier. Samples were subjected to mass spectrometry (MS) analysis. Peptides were analyzed using a Synapt High Definition Mass Spectrometer (Waters, UK) equipped with a nanoACQUITY Ultra Performance LC system (Waters, USA). In brief, $2 \mu \mathrm{l}$ of peptide solution was injected onto a $75 \mathrm{mx} 100 \mathrm{~mm}$ Atlantis dC18 column (Waters). Solvent A consisted of $0.1 \%$ formic acid in water, and solvent $\mathrm{B}$ was composed of $0.1 \%$ formic acid in ACN. Peptides were initially separated using $100 \mathrm{~min}$ gradients and electrosprayed into the mass spectrometer (fitted with a NanoLockSpray source) at a flow rate of $300 \mathrm{nl} / \mathrm{min}$. Mass spectra were obtained from m/z 300-1600 for $1 \mathrm{sec}$, followed by four data-dependent MS/MS scans from $\mathrm{m} / \mathrm{z}$ 50-1900 for $1 \mathrm{sec}$ each. The collision energy used to perform MS/MS was varied according to the mass and charge state of the eluting peptide. (Glu1)-Fibrinopeptide B was infused at a rate of $350 \mathrm{nl} / \mathrm{min}$, and an MS scan was obtained for $1 \mathrm{sec}$ every $30 \mathrm{sec}$ throughout the run. A database search was performed with MASCOT (Matrix Science, UK) using the following parameters: NCBInr.08.03.26 database, Rattus norvegicus species and maximum number of missed cleavage by trypsin at 1 . Mass tolerance ranged from \pm 50 to $100 \mathrm{ppm}$. The peptide modification allowed was carbamidomethylation in the fixed modification mode.

Statistical analysis. Data were shown as the mean \pm standard error of the mean (SEM). The PWL data were analyzed using two-way ANOVA followed by the Tukey test. Data from the quantitative analysis of differential phosphoproteins were analyzed using Kruskal-Wallis one-way ANOVA on ranks followed by the Student-Newman-Keuls test. $\mathrm{P}<0.05$ was considered to indicate a statistically significant difference.

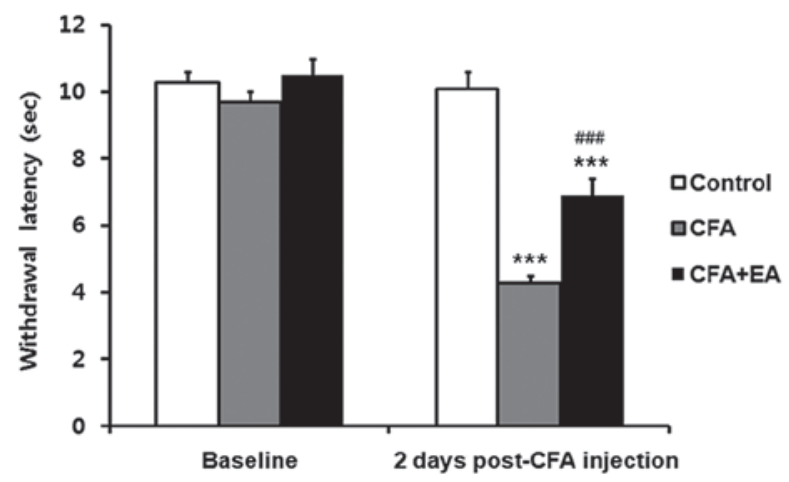

Figure 1. The effect of EA on CFA-induced inflammatory thermal hyperalgesia. Two days after the intraplantar injection of CFA, the mean PWL was significantly decreased compared to the baseline and the control group $(\mathrm{P}<0.001)$. Following EA treatment, $\mathrm{PWL}$ showed a significant improvement in comparison with CFA-induced inflammatory rats $(\mathrm{P}<0.001)$. The data are the mean \pm SEM ( $n=6$ in each group). ${ }^{* * *} \mathrm{P}<0.001$ indicates a significant difference compared to the control group (Control). ${ }^{\# \#} \mathrm{P}<0.001$ indicates a significant difference between the inflammatory pain group (CFA) and EA treatment group (CFA+EA). EA, electroacupuncture; CFA, complete Freund's adjuvant; SEM, standard error of the mean; PWL, paw withdrawal latency.

\section{Results}

Pain relief effect of EA on CFA-induced hyperalgesia. Prior to CFA injection, the overall mean baseline PWL to noxious heat stimuli showed no significant differences in all groups of rats. Following CFA injection, its latency was significantly decreased compared with that of baseline $(\mathrm{p}<0.001)$, whereas the saline injection did not change the PWL. Following EA treatment, however, the PWL showed significant improvement $(\mathrm{p}<0.001)$ compared with that of the CFA-injected inflammatory pain group (Fig. 1). These results suggest that EA stimulation may attenuate the CFA-induced inflammatory thermal hyperalgesia.

Differential phosphoprotein profiling between the inflammatory pain and EA treatment models. To investigate the alternative protein phosphorylation in the SCDH, protein extracts from the control, CFA-induced inflammatory pain and EA treatment groups were obtained and separated by 2-DE gel. Phosphoproteins were detected by Pro-Q Diamond staining and analyzed using SameSpot software (Fig. 2). Over 500 protein spots were detected on gels following Pro-Q Diamond staining, automatic spot detection, background subtraction and volume normalization. In three sets of comparison (control group vs. inflammatory pain group, control group vs. EA treatment group and inflammatory pain group vs. EA treatment group), the protein spots exhibiting significant changes of $>1.5$-fold changes were scored. In total, eight spots were selected as differentially phosphorylated proteins between the inflammatory pain group and the EA treatment group.

Identification of differentially phosphorylated proteins in the SCDH between the inflammatory pain and EA treatment models. Eight proteins that were differentially phosphorylated between the inflammatory and EA treatment groups were identified (Table I). Aldolase C, nascent polypeptide-associated 
Table I. List of phosphoproteins identified by mass spectrometry.

\begin{tabular}{|c|c|c|c|c|c|c|c|}
\hline \multirow[b]{2}{*}{ Spot no. } & \multirow[b]{2}{*}{ Accession no. } & \multirow[b]{2}{*}{ Protein } & \multirow[b]{2}{*}{ Abbreviation } & \multirow[b]{2}{*}{$\begin{array}{l}\text { Matched } \\
\text { peptides }\end{array}$} & \multirow[b]{2}{*}{$\begin{array}{l}\text { Moscot } \\
\text { score }\end{array}$} & \multirow[b]{2}{*}{$\begin{array}{l}\text { Coverage } \\
(\%)\end{array}$} & Fold change \\
\hline & & & & & & & $\mathrm{CFA}+\mathrm{EA}$ vs. CFA \\
\hline 16 & gil6978489 & Aldolase C & ALDOC & 21 & 654 & 47 & $3.4 \uparrow$ \\
\hline 21 & gil149029722 & $\begin{array}{l}\text { Nascent-polypeptide-associated } \\
\text { complex } \alpha\end{array}$ & NACA & 2 & 215 & 16 & $2.4 \uparrow$ \\
\hline 31 & gil20302113 & Stress-induced phosphoprotein 1 & STI1 & 24 & 632 & 40 & $2.4 \uparrow$ \\
\hline 36 & gil71534276 & GDP dissociation inhibitor 1 & GDI1 & 26 & 617 & 41 & $2.1 \downarrow$ \\
\hline 63 & gil28467005 & Heat shock protein 90 & Hsp90 & 17 & 467 & 22 & $1.8 \uparrow$ \\
\hline 82 & gil56090241 & Thiamine triphosphatase & THTPA & 9 & 254 & 27 & $1.6 \downarrow$ \\
\hline 84 & gil40254752 & Phosphoglycerate kinase 1 & PGK1 & 49 & 1379 & 69 & $1.6 \downarrow$ \\
\hline 109 & gil9507245 & $14-3-3 \gamma$ & YWHAG & 24 & 1025 & 65 & $1.5 \downarrow$ \\
\hline
\end{tabular}

The phosphorylation changes of identified proteins are indicated by $\uparrow$ (increase) and $\downarrow$ (decrease), with values indicating the fold changes of the proteins. CFA, complete Freund's adjuvant; EA, electroacupuncture.

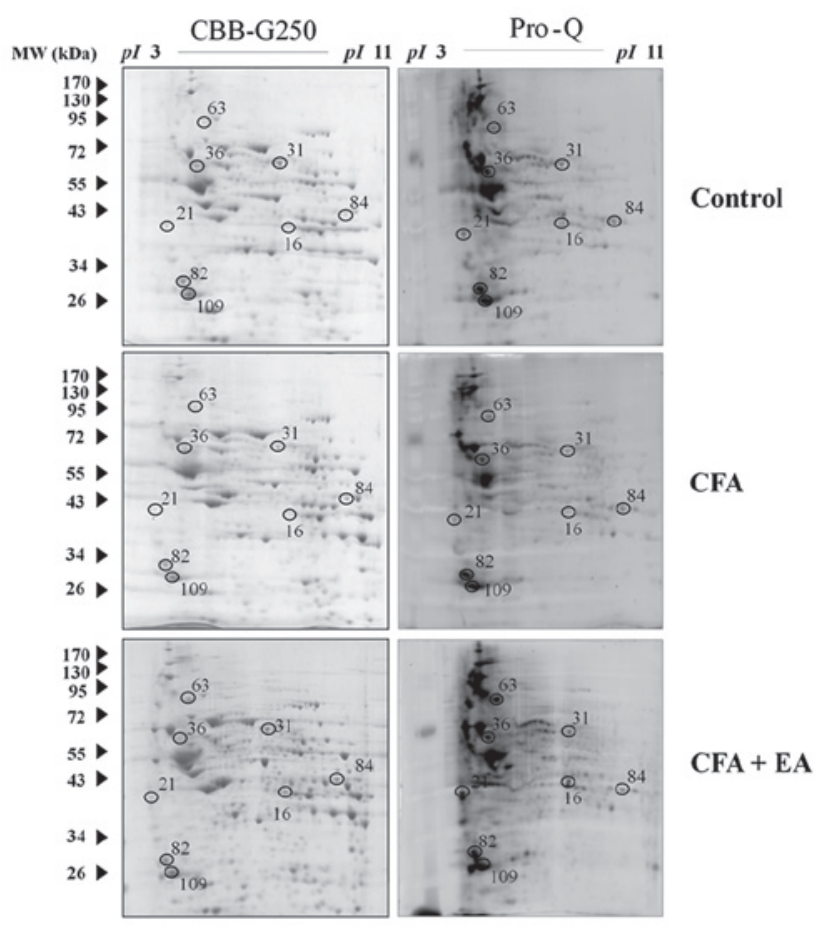

Figure 2. Representative 2-DE gel images of proteins from control, inflammatory pain model and EA treatment model rats. Total protein lysates were loaded on a 7-cm IPG strip with a non-linear $\mathrm{pH}$ gradient (pI 3-11) for isoelectric focusing, and separated by $13 \%$ SDS-PAGE. Proteins were stained with CBB-G250 and Pro-Q Diamond. Circles show the positions of eight identified protein spots. Spots were identified using liquid chromatographytandem mass spectrometry, as outlined in Table I. 2-DE, 2-dimensional gel electrophoresis; EA, electroacupuncture.

complex $\alpha$ (NACA), stress-induced phosphoprotein 1 (STI1) and heat shock protein 90 (Hsp90) proteins showed increased phosphorylation, whereas GDP dissociation inhibitor 1 (GDI1), thiamine triphosphatase, phosphoglycerate kinase 1 and 14-3-3 $\gamma$ proteins showed decreased phosphorylation levels following EA treatment in the inflammatory pain model. These proteins are involved in several key biological processes including signal transduction (14-3-3 $\gamma$, GDI1), chaperone function (STI1 and Hsp90), metabolism (aldolase C, phosphoglycerate kinase and thiamine triphosphatase) and protein transport (NACA).

Of the four proteins exhibiting increased phosphorylation in the EA treatment group compared to the inflammatory pain group, STI1 and Hsp90 showed decreased phosphorylation levels in the inflammatory pain model compared to control rats, and were restored to normal phosphorylation levels following EA treatment. Aldolase C and NACA did not show significant phosphorylation changes between the pain model and control rats, but EA treatment significantly increased the phosphorylation levels compared to the inflammatory pain group (Fig. 3).

Of the four proteins exhibiting decreased phosphorylation in the EA treatment group compared to the inflammatory pain group, thiamine triphosphatase showed significantly increased phosphorylation in the pain model compared to control rats, and EA treatment restored it to the control level. Three proteins including GDI1, phosphoglycerate kinase 1 and 14-3-3 $\gamma$ showed no significant changes between the pain model and control rats, but EA treatment significantly decreased phosphorylation levels compared to the inflammatory pain group (Fig. 3).

\section{Discussion}

The aim of the present study was to elucidate the EA analgesic mechanism from the aspect of protein phosphorylation changes in the SCDH using a phosphoproteomic approach. Since EA attenuated the thermal hyperalgesia of inflammatory pain rats at 2 days post-CFA injection, subsequent studies were performed to compare the protein phosphorylation changes in the SCDH of each experimental group at that time. Eight proteins were differentially phosphorylated following EA treatment in the inflammatory pain model. Although we did not detect low abundance proteins such as receptors and signaling kinases, which are involved in central sensitization 
A

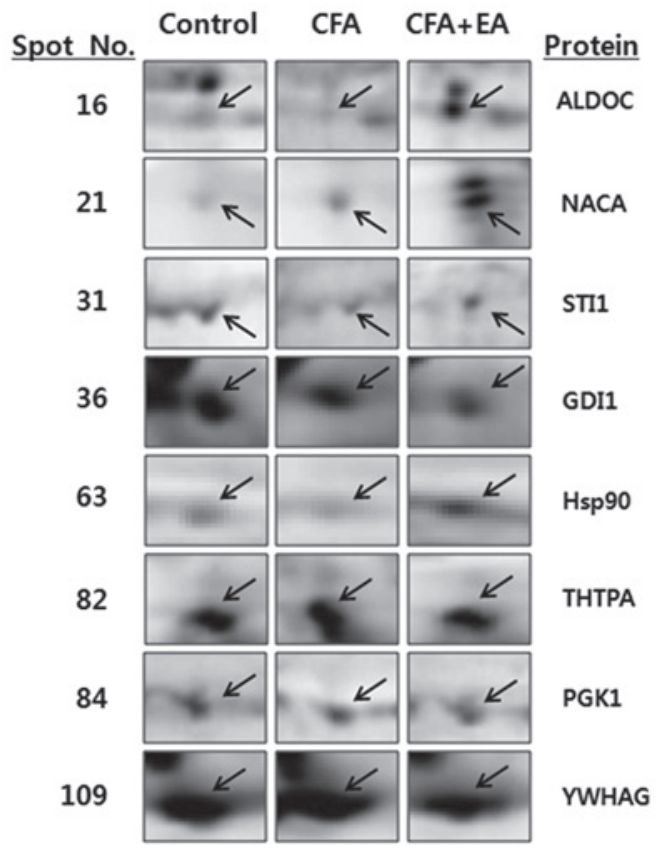

B

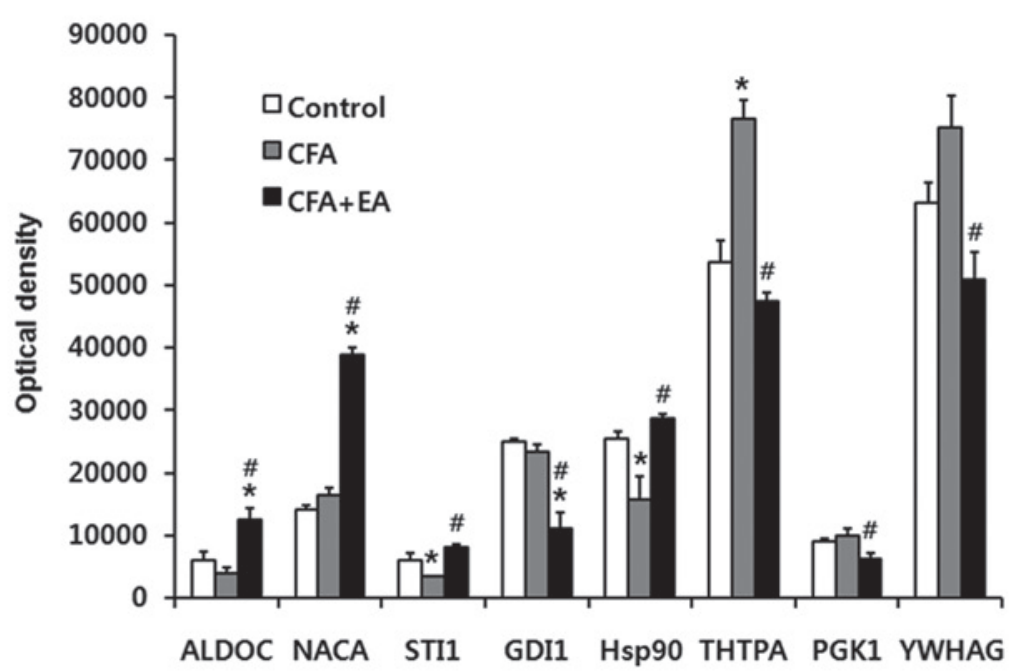

Figure 3. Expression profiles of eight proteins that were differentially phosphorylated between the inflammatory pain and EA treatment groups. (A) Magnified 2D gel map areas containing the identified spots. Arrows on the 2-DE gels are protein spots that showed significant phosphorylation changes between the inflammatory pain and EA treatment groups. (B) Quantification of relative spot densities of identified proteins. Data are the mean \pm SEM ( $=3$ in each group). ${ }^{*} \mathrm{P}<0.05$ indicates a significant difference compared to the control group (Control). ${ }^{*} \mathrm{P}<0.05$ indicates significant differences between the inflammatory pain group (CFA) and EA treatment group (CFA+EA). EA, electroacupuncture; CFA, complete Freund's adjuvant; SEM, standard error of the mean.

in the SCDH, we identified the novel proteins regulated by EA treatment that may be correlated with the EA analgesic mechanism.

It is notable that the phosphorylation levels of two molecular chaperones, STI1 and Hsp90, were decreased in the inflammatory pain model, but were recovered by EA stimulation. STI1 is a co-chaperone that associates with Hsp70 and Hsp90, and regulates the cell functions of these chaperones (17). STI1 is differentially expressed in the neuropathic rat pain model following spinal cord injury (18). Hsp90 is involved in microglial TLR4-mediated pain enhancement in rat SCDH (19). Although the effect of the phosphorylation of these proteins on inflammatory pain is unclear, our results suggest that there is a correlation between the phosphorylation level of chaperone proteins and inflammatory pain. In addition, our results demonstrate that EA changes the phosphorylation level of the chaperone proteins, indicating that it is may be involved in the EA analgesia.

GDP dissociation inhibitors (GDIs) block the dissociation of GDP from Rho proteins, maintaining the GTPase in an inactive form and preventing GTPase activation by guanine nucleotide exchange factors (GEFs). Rho GTPase acts as a molecular switch in a number of diverse cell processes including actin dynamics, gene transcription, cell-cycle progression and cell adhesion function. In particular, Rho GTPases are involved in the development of pain memory by modulating dendritic spine morphology and density in the $\operatorname{SCDH}(20,21)$. Several studies have shown that the phosphorylation of GDI is capable of inducing the dissociation of GDI from Rho GTPase, resulting in the activation of Rho GTPase (22-24). In the present study, the phosphorylation level of GDI1 was not changed in the inflammatory pain model compared to the control, but EA treatment decreased the phosphorylation level. Thus, EA may exert an analgesic effect by inhibiting Rho GTPase-regulated spine remodeling through the dephosphorylation of GDI1.

14-3-3- $\gamma$ belongs to the 14-3-3 family of proteins and is an adapter protein that is involved in a number of vital cell processes such as metabolism, protein trafficking, signal transduction, apoptosis and cell cycle regulation. Therefore, it is notable that $14-3-3-\gamma$ was identified as a differential protein. Previous proteomic studies reported that the expression levels of 14-3-3 proteins are altered in spinal cord injury pain models and EA treatment decreases the protein expression level $(18,25)$. In this study, EA stimulation was found to decrease the phosphorylation of 14-3-3 $\gamma$ protein compared with that of the inflammatory pain group, indicating that phosphorylation change of 14-3-3 proteins may be involved in EA analgesia.

Metabolic enzymes such as aldolase $\mathrm{C}$, phosphoglycerate kinase 1 and thiamine triphosphatase were also detected as differentially phosphorylated proteins. To respond to stress such as inflammatory pain, metabolic pathways that are inactive or active under normal state conditions need to be changed. In addition, a substantial amount of energy is required to make changes in the protein expression and to build up cell components. Therefore, it is conceivable that several metabolic enzymes were detected in the present study. Aldolase $\mathrm{C}$ is also differentially expressed in a number of neuropathic pain models (25-27). Therefore, our data suggest that the regulation of metabolic enzymes are involved in pain development and the analgesic effects of EA. 
NACA is a cytosolic protein that binds nascent-polypeptide domains emerging from the ribosome and assists in the post-translational process. In addition to acting as a protein translation chaperone, NACA also functions as a transcriptional coactivator in conjunction with an acidic activator $(28,29)$. NACA may be phosphorylated by several kinases in vitro and its function as a transcriptional coactivator is regulated by phosphorylation change $(30,31)$. Findings of the present study have shown that the phosphorylation level of NACA was not altered in the inflammatory pain model compared to the control, but that EA treatment increased the phosphorylation level. Although the effect of phosphorylation on inflammatory pain is unclear, our results suggest that the phosphorylation change of NACA may be involved in EA analgesia.

In conclusion, we identified differentially phosphorylated proteins between inflammatory pain and EA treatment models using phosphoproteomic analysis. These proteins may be involved in the EA analgesic mechanism by regulating cellular processes such as signal transduction, chaperone function, metabolic change, protein transport and transcription.

\section{Acknowledgements}

This study was supported by grants from KRIBB Initiative Research Program (to K-H. Bae) and Mid-career Research Program (to K.-H. Bae, Grant no. 2010-0022319).

\section{References}

1. Latremoliere A and Woolf CJ: Central sensitization: a generator of pain hypersensitivity by central neural plasticity. J Pain 10: 895-926, 2009.

2. Woolf CJ: Central sensitization: uncovering the relation between pain and plasticity. Anesthesiology 106: 864-867, 2007.

3. SvenssonCI,Marsala M,Westerlund A, Calcutt NA,CampanaWM, Freshwater JD, Catalano R, Feng Y, Protter AA, Scott B and Yaksh TL: Activation of p38 mitogen-activated protein kinase in spinal microglia is a critical link in inflammation-induced spinal pain processing. J Neurochem 86: 1534-1544, 2003.

4. Ji RR, Gereau RW IV, Malcangio M and Strichartz GR: MAP kinase and pain. Brain Res Rev 60: 135-148, 2009.

5. Gao YJ, Xu ZZ, Liu YC, Wen YR, Decosterd I and Ji RR: The c-jun N-terminal kinase 1 (JNK1) in spinal astrocytes is required for the maintenance of bilateral mechanical allodynia under a persistent inflammatory pain condition. Pain 148: 309-319, 2010

6. Ji RR, Befort K, Brenner GJ and Woolf CJ: ERK MAP kinase activation in superficial spinal cord neurons induces prodynorphin and NK-1 upregulation and contributes to persistent inflammatory pain hypersensitivity. J Neurosci 22: 478-485, 2002.

7. Seybold VS, Jia YP and Abrahams LG: Cyclo-oxygenase-2 contributes to central sensitization in rats with peripheral inflammation. Pain 105: 47-55, 2003.

8. Zhang YQ, Ji GC, Wu GC and Zhao ZQ: Excitatory amino acid receptor antagonists and electroacupuncture synergetically inhibit carrageenan-induced behavioral hyperalgesia and spinal fos expression in rats. Pain 99: 525-535, 2002.

9. Sun S, Chen WL, Wang PF, Zhao ZQ and Zhang YQ: Disruption of glial function enhances electroacupuncture analgesia in arthritic rats. Exp Neurol 198: 294-302, 2006.

10. Shan S, Qi-Liang MY, Hong C, Tingting L, Mei H, Haili P, Yan-Qing W, Zhi-Qi Z and Yu-Qiu Z: Is functional state of spinal microglia involved in the anti-allodynic and anti-hyperalgesic effects of electroacupuncture in rat model of monoarthritis? Neurobiol Dis 26: 558-568, 2007.

11. Choi BT, Kang J and Jo UB: Effects of electroacupuncture with different frequencies on spinal ionotropic glutamate receptor expression in complete Freund's adjuvant-injected rat. Acta Histochem 107: 67-76, 2005.

12. Raggiaschi R, Gotta S and Terstappen GC: Phosphoproteome analysis. Biosci Rep 25: 33-44, 2005.
13. Reinders J and Sickmann A: State-of-the-art in phosphoproteomics. Proteomics 5: 4052-4061, 2005.

14. Kalume DE, Molina H and Pandey A: Tackling the phosphoproteome: tools and strategies. Curr Opin Chem Biol 7: 64-69, 2003.

15. Stein C, Millan MJ and Herz A: Unilateral inflammation of the hindpaw in rats as a model of prolonged noxious stimulation: alterations in behavior and nociceptive thresholds. Pharmacol Biochem Behav 31: 445-451, 1988.

16. Lao L, Zhang G, Wei F, Berman BM and Ren K: Electroacupuncture attenuates behavioral hyperalgesia and selectively reduces spinal fos protein expression in rats with persistent inflammation. J Pain 2: 111-117, 2001.

17. Odunuga OO, Longshaw VM and Blatch GL: Hop: more than an Hsp70/Hsp90 adaptor protein. Bioessays 26: 1058-1068, 2004.

18. Afjehi-Sadat L, Brejnikow M, Kang SU, Vishwanath V, Walder N, Herkner K, Redl H and Lubec G: Differential protein levels and post-translational modifications in spinal cord injury of the rat. J Proteome Res 9: 1591-1597, 2010.

19. Hutchinson MR, Ramos KM, Loram LC, Wieseler J, Sholar PW, Kearney JJ, Lewis MT, Crysdale NY, Zhang Y, Harrison JA, Maier SF, Rice KC and Watkins LR: Evidence for a role of heat shock protein-90 in toll like receptor 4 mediated pain enhancement in rats. Neuroscience 164: 1821-1832, 2009.

20. Fu Q, Hue J and Li S: Nonsteroidal anti-inflammatory drugs promote axon regeneration via RhoA inhibition. J Neurosci 27: 4154-4164, 2007.

21. Tan AM, Stamboulian S, Chang YW, Zhao P, Hains AB, Waxman SG and Hains BC: Neuropathic pain memory is maintained by Rac1-regulated dendritic spine remodeling after spinal cord injury. J Neurosci 28: 13173-13183, 2008.

22. DerMardirossian C and Bokoch GM: GDIs: central regulatory molecules in rho GTPase activation. Trends Cell Biol 15: 356-363, 2005.

23. DerMardirossian C, Schnelzer A and Bokoch GM: Phosphorylation of RhoGDI by Pak1 mediates dissociation of Rac GTPase. Mol Cell 15: 117-127, 2004.

24. Qiao J, Holian O, Lee BS, Huang F, Zhang J and Lum H: Phosphorylation of GTP dissociation inhibitor by PKA negatively regulates RhoA. Am J Physiol Cell Physiol 295: C1161-C1168, 2008.

25. Sung HJ, Kim YS, Kim IS, Jang SW, Kim YR, Na DS, Han KH, Hwang BG, Park DS and Ko J: Proteomic analysis of differential protein expression in neuropathic pain and electroacupuncture treatment models. Proteomics 4: 2805-2813, 2004.

26. Jimenez CR, Stam FJ, Li KW, Gouwenberg Y, Hornshaw MP, De Winter F, Verhaagen J and Smit AB: Proteomics of the injured rat sciatic nerve reveals protein expression dynamics during regeneration. Mol Cell Proteomics 4: 120-132, 2005.

27. Komori N, Takemori N, Kim HK, Singh A, Hwang SH, Foreman RD, Chung K, Chung JM and Matsumoto H: Proteomics study of neuropathic and nonneuropathic dorsal root ganglia: altered protein regulation following segmental spinal nerve ligation injury. Physiol Genomics 29: 215-230, 2007.

28. Rospert S, Dubaquie Y and Gautschi M: Nascent-polypeptideassociated complex. Cell Mol Life Sci 59: 1632-1639, 2002.

29. Yotov WV, Moreau A and St-Arnaud R: The alpha chain of the nascent polypeptide-associated complex functions as a transcriptional coactivator. Mol Cell Biol 18: 1303-1311, 1998.

30. Quelo I, Akhouayri O, Prud'homme J and St-Arnaud R: GSK3 beta-dependent phosphorylation of the alpha NAC coactivator regulates its nuclear translocation and proteasome-mediated degradation. Biochemistry 43: 2906-2914, 2004.

31. Quelo I, Gauthier C, Hannigan GE, Dedhar S and St-Arnaud R: Integrin-linked kinase regulates the nuclear entry of the c-jun coactivator alpha-NAC and its coactivation potency. J Biol Chem 279: 43893-43899, 2004. 